\title{
Efficacy of Para-Aortic Lymphadenectomy in Ovarian Cancer: A Retrospective Study
}

\author{
Hiromi Ugaki ${ }^{*}$, Yosiko Komoto $^{1}$, Reisa Kakubari ${ }^{1}$, Eriko Tanaka $^{1}$, Hisashi Konishi ${ }^{1}$, Toshihiro Kitai ${ }^{1}$, \\ Saori Nakajima ${ }^{1}$, Miho Muraji ${ }^{1}$, Takayuki Enomoto ${ }^{2}$, Masahiko Takemura ${ }^{1}$ \\ ${ }^{1}$ Department of Obstetrics and Gynecology, Osaka General Medical Center, Osaka, Japan; ${ }^{2}$ Department of Obstetrics and Gynecol- \\ ogy, Niigata University Graduate School of Medical and Dental Science, Niigata, Japan. \\ Email: *hirohiromimi@hotmail.com
}

Received March 21 ${ }^{\text {st }}$ 2013; revised April 23 $3^{\text {rd }}$, 2013; accepted May $3^{\text {rd }}, 2013$

Copyright (C) 2013 Hiromi Ugaki et al. This is an open access article distributed under the Creative Commons Attribution License, which permits unrestricted use, distribution, and reproduction in any medium, provided the original work is properly cited.

\begin{abstract}
Objective: The prognostic impact for ovarian cancer treatment of employing a systematic para-aortic and pelvic lymphadenectomy is still poorly defined. The purpose of this study was to evaluate the therapeutic efficacy of adding a para-aortic lymphadenectomy (PA) to the pelvic lymphadenectomy (PL), as compared with solely the pelvic lymphadenectomy. Materials and Methods: A retrospective study of patient outcomes was conducted of ovarian cancer patients who underwent optimal debulking surgery, concurrent with either PA + PL or PL alone, between 2000 and 2009 at our Osaka General Medical Center. Results: One hundred twenty-one patients with ovarian cancer underwent surgery. Forty-four patients (36\%) underwent optimal debulking surgery (all residual disease was $<1 \mathrm{~cm}$ ) concurrent with lymphadenectomy. Seventeen patients underwent PA + PL (PA group), and 27 patients underwent PL alone (PL group). There were no significant differences in terms of overall survival (OS; hazard ratio [HR] $=0.49 ; 95 \%$ CI, 0.13 to 1.82; $p=0.29$ ) and progression-free survival (PFS; $\mathrm{HR}=0.62 ; 95 \% \mathrm{CI}, 0.19$ to $2.00 ; p=0.40$ ) between the PA group and the PL group. Both OS and PFS also failed to show significant differences, even when comparing them among 26 cases of FIGO stage I cases. Conclusions: Our data failed to show any prognostic improvement for ovarian cancer by adding para-aortic lymphadenectomy to the standard pelvic lymphadenectomy regimen.
\end{abstract}

Keywords: Ovarian Cancer; Pelvic Lymphadenectomy; Para-Aortic Lymphadenectomy

\section{Introduction}

Ovarian cancer has been increasing in Japan. Approximately 8000 new ovarian cancer cases, and 4500 ovarian cancer deaths, were recorded in 2006 [1]. Retroperitoneal lymph nodes involvement occurs in approximately $50 \%$ to $80 \%$ of women with advanced ovarian cancer [2]. Cass et al. found that $15 \%$ of patients with clinical stage I disease have microscopic lymph node metastases [3]. In recognition of the prognostic importance of the retroperitoneal spread of ovarian cancer, the FIGO staging classification was amended in 1988 to include a substage for nodal involvement [4]. Subsequent work has illuminated the relevant surgical anatomy, which has allowed for identification of the role and technical aspects of lymph node dissection, and for a clarification of the nomenclature [5-7].

Primary cytoreductive surgery (i.e., the removal of as

${ }^{*}$ Corresponding author. much of the tumor as possible at the time of initial surgery, with resection of only the bulky nodes) has been an integral part of the treatment of advanced ovarian cancer. In addition, the amount of postoperative residual tumor is a clinically significant prognostic factor [8,9].

It is still unclear as to whether the systematic removal of the retroperitoneal para-aortic lymph nodes should be a standard part of a maximal cytoreductive surgery. The core issue of the controversy is whether or not the removal of these lymph nodes improves patient survival.

The purpose of this study was to evaluate the therapeutic efficacy of para-aortic lymphadenectomy (PA) being added to the pelvic lymphadenectomy (PL), as compared with a pelvic lymphadenectomy alone.

\section{Materials and Methods}

A retrospective review of the medical records of the Osaka General Medical Center (Osaka, Japan) was con- 
ducted for the period between January 1, 2000 and December 31, 2009 to identify all patients diagnosed as primary ovarian cancer who were treated with primary surgery. Patients with borderline tumors were excluded from this study.

The primary surgery was aimed at removing the primary tumor and visible pelvic implants. This included treatment with total abdominal hysterectomy and bilateral salpingo-oophorectomy and/or omentectomy and/or appendectomy, before adding the lymphadenectomy. As a consequence to this surgery, participants had no visible residual tumors greater than $1 \mathrm{~cm}$ in diameter. The extent of the lymph node dissection included a pelvic lymphadenectomy without (PL) or with (PA) a para-aortic lymphadenectomy.

The indication for preoperatively being put in the PL group was that para-aortic lymph node metastases which lymph node size is less than $1 \mathrm{~cm}$ in diameter were not found by Computed Tomography (CT) or Magnetic Resonance Imaging (MRI).

Pelvic lymphadenectomy: The dissection was begun at the origin of the external iliac vessels and continued caudally around them along the medial border of the psoas muscle; the lower limit of the external iliac lymphadenectomy was represented by the deep inferior epigastric vessels. The lateral boundaries of dissection were superficially delineated by the fascia covering the psoas muscle and deeply by the fascia covering the internal obturator and levator ani muscles; the median margin of the lymphadenectomy was represented by an imaginary plane which was parallel to the umbilical artery and was delineated by the umbelico-pubic fascia, the bladder and the rectum. In addition, lymphatic tissue was cleared from the obturator fossa, which was begun with the mobilizations of the superficial obturator nodes, which were removed en bloc with the lymphatic fatty tissue, which has been previously separated from the internal iliac vessels to the origin of the internal pudendal vessels.

Aortic lymphadenectomy: The nodal dissection started at the aortic bifurcation by removing the superficial intercavoaortic, precaval and preaortic nodal groups. Then lymph nodes located lateral to the cava (paracaval) were separated from the vena cava, the renal capsule and the psoas muscle, and were then removed en bloc. Afterward, displacing the vena cava and the aorta laterally and medially, the lymph nodes behind the cava (retrocaval nodal group) and the lumbar vessels (deep intercavoaortic nodes) were separated from the prevertebral fascia and then removed. Removal of the most cranial nodes, both behind and under the left renal vein, was performed after entering the right plane of dissection between the Toldt's and Gerota's fasciae, mobilizing the descending colon from the renal capsule, the psoas, the ovarian pedicle and the ureter, and displacing it medially.
The patients received from 3 to 6 cycles of paclitaxel at a dose of $175 \mathrm{mg}$ per square meter of body-surface area, and carboplatin at a dose of area under the curve (AUC) $5 \mathrm{mg} / \mathrm{mL}$ every three weeks for adjuvant chemotherapy for FIGO stages Ic, II, III and IV.

A statistical analysis for demographic characteristics of the groups was performed using the Mann-Whitney U test. Overall survival was calculated from the day of first surgical treatment until death, regardless of the cause of death. Progression-free survival was calculated from the day of surgical treatment to the time of either detected progression or death. Overall-survival curves and progression-free survival curves were calculated for each treatment group using Kaplan-Meier estimates and were compared with the log-rank test $[10,11]$. $p$ values of less than 0.05 were considered to be statistically significant.

\section{Results}

Between 2000 and 2009, 121 women with ovarian cancer had undergone surgical treatment at our facility. Fortyfour patients (36\%) underwent optimally debulked surgery (maximum size of the residual disease $<1 \mathrm{~cm}$ ) concurrent with some level of lymphadenectomy. Seventeen patients underwent PA + PL (PA group) and 27 patients underwent PL alone (PL group).

The details of the patient's characteristics are shown in Table 1. The mean ages were 60 and 59 years for the PA and PL groups, respectively ( $p=0.5225)$. BMI [body mass index] were 23 for PA group and 21 for PL group, respectively ( $p=0.779$ ). There were no significant difference is outward patient characteristics between the PA group and the PL group. Blood loss volume was significantly higher in PA group compared with the PL group ( $p=0.0266$ ); whereas the surgical time was not different between these two groups.

Progression-free 5-year survival was 69\% for the PA group and 75\% for PL group (Figure 1). Overall 5-year survival was $71 \%$ for the PA group and $90 \%$ for the PL group (Figure 2). Comparing the patients in the PA group with the PL group, there was no significant difference in overall survival (OS; hazard ratio [HR] $=0.49$; $95 \% \mathrm{CI}, 0.13$ to $1.82 ; p=0.29$ ) or in progression-free survival $(\mathrm{PFS}$; HR $=0.62 ; 95 \% \mathrm{CI}, 0.19$ to $2.00 ; p=$ 0.40).

Additionally, we analyzed the FIGO stage I of 26 patients. Eight women from the PA group and 17 women from the PL group participated. Progression-free 5-year survival was $75 \%$ for the PA group and $79 \%$ for the PL group (Figure 3). Overall 5-year survival was 75\% for the PA group and 93\% for the PL group (Figure 4). There were no significant differences between the PA group and the PL group in OS (HR $=0.62 ; 95 \% \mathrm{CI}, 0.08$ to $4.68 ; p=0.63$ ) or PFS (HR $=0.66$; $95 \% \mathrm{CI}, 0.10$ to 
Table 1. Patient characteristics.

\begin{tabular}{|c|c|c|c|}
\hline & PA group $(\mathrm{n}=17)$ & PL group $(n=27)$ & $p$ \\
\hline Mean age $\left(25^{\text {th }}-75^{\text {th }}\right.$ percentiles $)$ & $60(53-67)$ & $59(46-67)$ & 0.5225 \\
\hline BMI $\left(25^{\text {th }}-75^{\text {th }}\right.$ percentiles $)$ & $23(20-25)$ & $21(20-25)$ & 0.779 \\
\hline \multicolumn{4}{|l|}{ Stage } \\
\hline I & 8 & 18 & \\
\hline II & 1 & 5 & \\
\hline III & 7 & 4 & \\
\hline IV & 1 & 0 & \\
\hline \multicolumn{4}{|l|}{ Histology } \\
\hline serous & 10 & 4 & \\
\hline mucinous & 1 & 6 & \\
\hline endometrioid & 0 & 3 & \\
\hline clear & 3 & 8 & \\
\hline mixed & 1 & 2 & \\
\hline mature teratoma with malignant change & 0 & 2 & \\
\hline carcinosarcoma & 1 & 1 & \\
\hline transitional cell carcinoma & 0 & 1 & \\
\hline granulosa & 1 & 0 & \\
\hline \multicolumn{4}{|l|}{ surgical data } \\
\hline mean operative time (min) & 257 & 232 & 0.3987 \\
\hline mean operative blood loss (ml) & 825 & 540 & 0.0266 \\
\hline
\end{tabular}

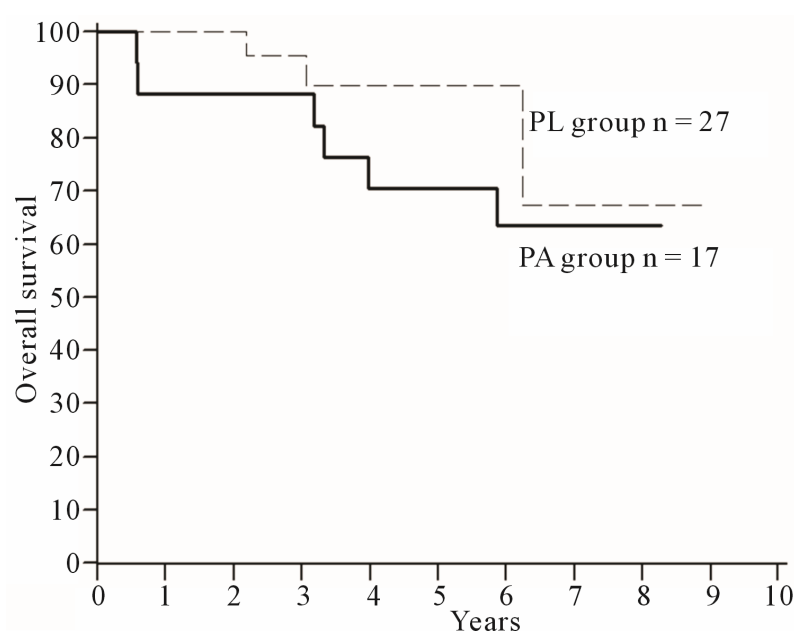

Figure 1. Overall survival curves for the PA group versus the PL group in ovarian cancer.

4.45; $p=0.65)$. Overall, the PA group tended to have a lower survival rate than the PL group.

In cases with recurrence, which we show in Table 2, only one out of the six cases was found to have para-

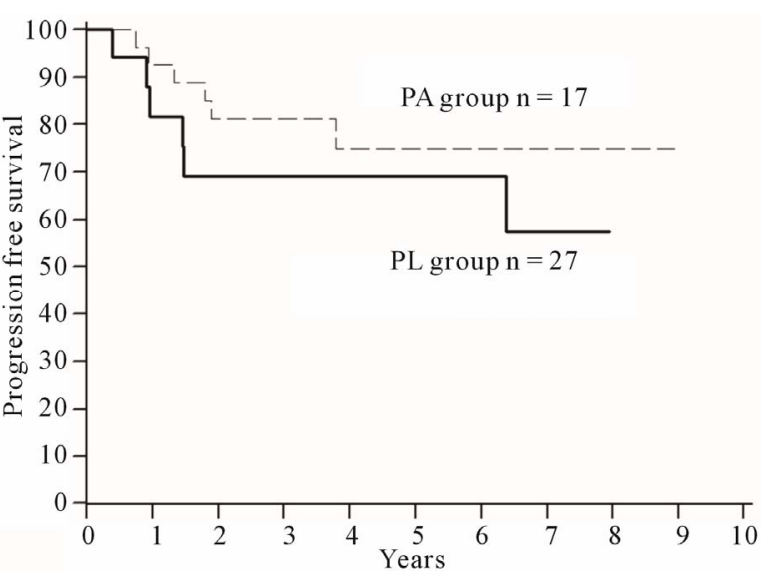

Figure 2. Progression-free survival curves for the PA group versus the PL group in ovarian cancer.

aortic lymph node recurrence, and this was in the PL group.

\section{Discussion}

Standard therapy for women with ovarian cancer in 


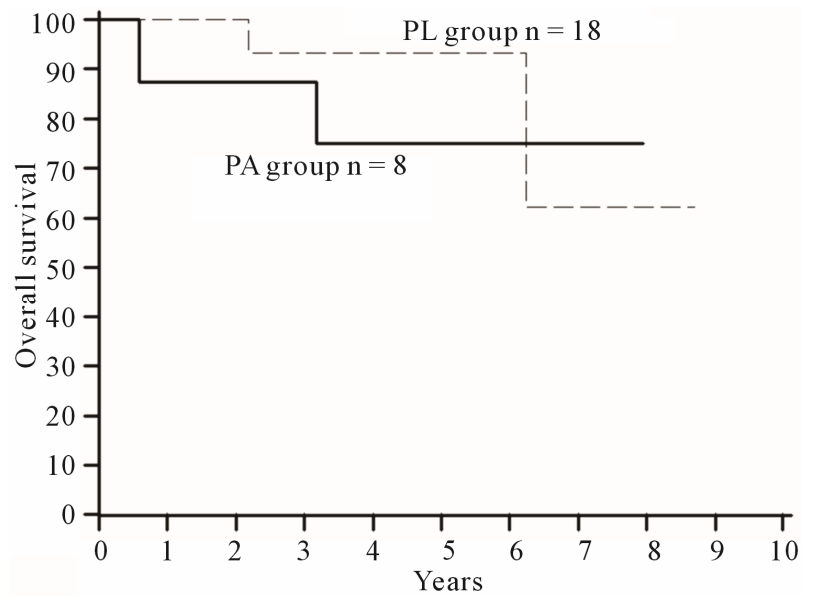

Figure 3. Overall survival curves for the PA group versus the PL group in stage I ovarian cancer.

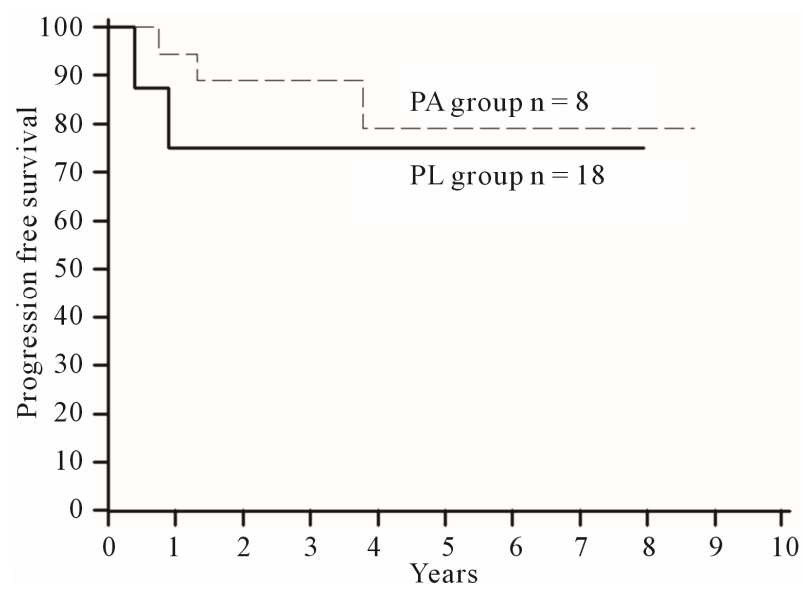

Figure 4. Progression-free survival curves for the PA group versus the PL group in stage I ovarian cancer.

Table 2. Portion of initial recurrence.

\begin{tabular}{cc}
\hline PA group & \\
Case 1 & stump \\
Case 2 & liver \\
Case 3 & peritoneum \\
Case 4 & peritoneum \\
Case 5 & lung \\
Case 6 & increase tumor maker \\
PL group & liver \\
Case 7 & peritoneum \\
Case 8 & para-aortic lymph node \\
Case 9 & inguinal lymph node \\
Case 10 & liver \\
Case 11 & outside of colon splenic flexure \\
Case 12 &
\end{tabular}

cludes a staging procedure with lymphadenectomy [4,12]. As patients with metastases to the lymph nodes have poorer outcomes, lymphadenectomy plays a significant diagnostic role in assessing a prognosis and determining their need for adjuvant treatment.

However, there are only a very limited number of studies which have investigated the therapeutic efficacy of adding on a dissection of the para-aortic lymph nodes to the traditional pelvic lymphadenectomy. There are even fewer studies which examined the benefits of lymphadenectomy in those patients in the earliest stages of ovarian cancer. Furthermore, there are risks of developing post-lymphadenectomy complications, such as ileus, deep-vein thrombosis, lymphocyst, lymphedema and major wound dehiscence. Additional complications potentially arising from systematic lymphadenectomy can be longer operating times and higher blood loss volumes than for a non-systematic lymphadenectomy [13,14].

Bristow et al. confirmed that, in FIGO stages III - IV ovarian cancer, a maximal surgical cytoreduction which included a systemic lymphadenectomy was one of the most powerful determinants of cohort survival [15]. Likewise, Onda et al. found that the overall metastasis-positive rates of the aortic and pelvic lymph nodes in all clinical stages were $38 \%$ and $37 \%$, respectively. They also found that $15 \%$ of lymph-node-positive patients with stage I/II tumors had only isolated aortic lymph node involvement, and another $23 \%$ had isolated pelvic lymph node involvement, clearly showing that direct spreading solely to the aortic or pelvic lymph nodes is possible in ovarian cancer. Moreover, they found that the incidences of positive lymph nodes in stages I and II ovarian carcinoma were approximately $20 \%$ in each. Stage I and stage II serous and clear cell types had significantly higher rates than endometrioid and mucinous types of the same stage [16].

A randomized study [14] showed that: 1) the addition of systematic aortic and pelvic lymphadenectomy to cytoreductive surgery prolonged progression-free survival, which, in turn, may have an important impact on the quality of life of patients with advanced ovarian cancer; 2) systematic lymphadenectomy did not prolong overall survival, probably because effective platinum based firstand second-line (with or without salvage surgery) chemotherapies might have diluted the impact of systematic lymphadenectomy on the risk of death; 3) patients in the systematic lymphadenectomy arm had a higher number of nodal metastases than patients in the no-lymphadenectomy arm. This study found that, although systematic lymphadenectomy significantly improved progression-free survival, overall survival was similar in both the systematic lymphadenectomy and the "resection of bulky nodes only" groups.

It is unknown if patients who underwent an aortic lym- 
phadenectomy may have received more chemotherapy, resulting in a better survival. Our data could not show any improvement of either PFS or OS by adding aortic lymphadenectomy to a pelvic lymphadenectomy. Furthermore, considering the chances of para-aortic lymph node metastases, cases diagnosed as FIGO stage I without para-aortic lymphadenectomy may have actually contain undiagnosed FIGO stage III cases.

We could not show any therapeutic effect of performing a para-aortic lymphadenectomy, even in the FIGO stage I case containing potential FIGO stage III case. The number of participants in this study is acknowledged to be able to provide only a limited reliability for the results. However, we believe our data do give adequate validity for considering doing a prospective study to evaluate the efficacy of aortic lymphadenectomy.

\section{REFERENCES}

[1] T. Matsuda, T. Marugame, K. I. Kamo, et al., “The Japan Cancer Surveillance Research Group. Cancer Incidence and Incidence Rates in Japan in 2005: Based on Data from 12 Population-Based Cancer Registries in the Monitoring of Cancer Incidence in Japan (MCIJ) Project," Japanese Journal of Clinical Oncology, Vol. 41, No. 1, 2011, pp. 139-147. doi:10.1093/jico/hyq169

[2] E. Burghardt, F. Girardi, M. Lahousen, et al., "Patterns of Pelvic and Paraaortic Lymph Node Involvement in Ovarian Cancer,” Gynecologic Oncology, Vol. 40, No. 2, 1991, pp. 103-106. doi:10.1016/0090-8258(91)90099-Q

[3] I. Cass, A. J. Li, C. D. Runowicz, et al., "Pattern of Lymph Node Metastases in Clinically Unilateral Stage I Invasive Epithelial Ovarian Carcinomas," Gynecologic Oncology, Vol. 80, No. 1, 2001, pp. 56-61. doi:10.1006/gyno.2000.6027

[4] FIGO Cancer Committee, "Staging Announcements," Gynecologic Oncology, Vol. 25, No. 3, 1986, pp. 383-385. doi:10.1016/0090-8258(86)90092-2

[5] P. B. Panici, G. Scambia, G. Baiocchi, et al., "Anatomical Study of Para-Aortic and Pelvic Lymph-Nodes in Gynecologic Malignancies,” Obstetrics \& Gynecology, Vol. 79, No. 4, 1992, pp. 498-502.

[6] G. Ferraris, A. Lanza, F. D’Addato, et al., “Techniques of Pelvic and Para-Aortic Lymphadenectomy in the Surgical Treatment of Cervix Carcinoma," European Journal of Gynecologic Oncology, Vol. 9, 1998, pp. 83-86.
[7] P. B. Panici, G. Scambia, G. Baiocchi, et al., “Technique and Feasibility of Systematic Paraaortic and Pelvic Lymphadenectomy in Gynecologic Malignancies, A Prospective Study," International Journal of Gynecological Cancer, Vol. 1, No. 3, 1991, pp. 133-140. doi:10.1111/j.1525-1438.1991.tb00028.x

[8] C. T. Griffiths, "Surgical Resection of Tumor Bulk in the Primary Treatment of Ovarian Carcinoma,” The Journal of the National Cancer Institute, Vol. 42, 1975, pp. 101104.

[9] N. F. Hacker, J. S. Berek, L. D. Lagasse, et al., "Primary Cytoreductive Surgery for Epithelial Ovarian Cancer," Obstetrics \& Gynecology, Vol. 61, No. 4, 1983, pp. 413420

[10] E. L. Kaplan and P. Meier, "Non Parametric Estimation from Incomplete Observations," Journal of the American Statistical Association, Vol. 53, No. 282, 1958, pp. $457-$ 481. doi:10.1080/01621459.1958.10501452

[11] R. Peto, M. C. Pike, P. Armitage, et al., "Design and Analysis of Randomized Clinical Trials Requiring Prolonged Observation of Each Patient. II. Analysis and Example,” British Journal of Cancer, Vol. 35, No. 1, 1977, pp. 1-39. doi:10.1038/bjc.1977.1

[12] P. J. Di Saia and W. T. Creasman, "Clinical Gynecologic Oncology,” 6th Edition, Mosby, St. Louis, 2002.

[13] ASTEC Study Group, H. Kitchener, A. M. Swart, Q. Qian, et al., "Efficacy of Systematic Pelvic Lymphadenectomy in Endometrial Cancer (MRC ASTECtrial): A Randomized Study,” Lancet, Vol. 373, No. 9658, 2009, pp. 125-136.

[14] P. B. Panici, A. Maggioni, N. Hacker, et al., "Systematic Aortic and Pelvic Lymphadenectomy versus Resection of Bulky Nodes only in Optimally Debulked Advanced Ovarian Cancer: A Randomized Clinical Trial," The Journal of the National Cancer Institute, Vol. 97, No. 8, 2005, pp. 560-566. doi:10.1093/jnci/dji102

[15] R. E. Bristow, R. S. Tomacruz, D. K. Armstrong, et al., "Survival Effect of Maximal Cytoreductive Surgery for Advanced Ovarian Carcinoma during Platinum Era: A Meta-Analysis,” Journal of Clinical Oncology, Vol. 20, No. 5, 2002, pp. 1248-1259. doi:10.1200/JCO.20.5.1248

[16] T. Onda, H. Yoshikawa, H. Yokota, et al., "Assessment of Metastases to Aortic and Pelvic Lymph Nodes in Epithelial Ovarian Carcinoma. A Proposal for Essential Sites for Lymph Node Biopsy,” Cancer, Vol. 78, No. 4, 1996, pp. 803-808. doi:10.1002/(SICI)1097-0142(19960815)78:4<803::AIDCNCR17>3.0.CO;2-Z 